\title{
The technological obsolescence of the Brazilian eletronic ballot box
}

\author{
Carlos Rogério Camargo ${ }^{\mathrm{a}^{*}}$, Richard Faust ${ }^{\mathrm{b}}$, Eugênio Merino ${ }^{\mathrm{a}}$ and Clarissa Stefani ${ }^{\mathrm{a}}$ \\ ${ }^{a}$ Departamento de Engenharia de Produção, Universidade Federal de Santa Catarina \\ ${ }^{b}$ Departamento de Engenharia do Conhecimento, Universidade Federal de Santa Catarina
}

\begin{abstract}
The electronic ballot box has played a significant role in the consolidation of Brazilian political process. It has enabled paper ballots extinction as a support for the elector's vote as well as for voting counting processes. It is also widely known that election automation has decisively collaborated to the legitimization of Brazilian democracy, getting rid of doubts about the winning candidates. In 1995, when the project was conceived, it represented a compromise solution, balancing technical efficiency and costs trade-offs. However, this architecture currently limits the ergonomic enhancements to the device operation, transportation, maintenance and storage. Nowadays are available in the market devices of reduced dimensions, based on novel computational architecture, namely tablet computers, which emphasizes usability, autonomy, portability, security and low power consumption. Therefore, the proposal under discussion is the replacement of the current electronic ballot boxes for tablet-based devices to improve the ergonomics aspects of the Brazilian voting process. These devices offer a plethora of integrated features (e.g., capacitive touchscreen, speakers, microphone) that enable highly usable and simple user interfaces, in addition to enhancing the voting process security mechanisms. Finally, their operational systems features allow for the development of highly secure applications, suitable to the requirements of a voting process.
\end{abstract}

Keywords: Public Elections; Political Process; Usability; Portability; Security.

\section{Introduction}

The electronic ballot box has played a significant role in the consolidation of Brazilian political process. It has enabled paper ballots extinction as a support for the elector's vote as well as for voting counting processes. It is also widely known that election automation has decisively collaborated to the legitimization of Brazilian democracy, getting rid of doubts about the winning candidates.

Consequently, it has eliminated objectionable human intervention in critical elections activities, preventing mistakes and, consequently, frauds. On the other hand, the simplicity of voting, including the identification of candidates and parties by numbers, has appreciably reduced the amount of votes that used to be canceled due to the impossibility to interpret what had been written by the voter on the paper ballots. Thus, the elections automation has increased the citizens' effective participation in the political process, and has definitely helped legitimize Brazilian democracy, eliminating doubts about the winning candidates.

However, if analyzed under a technological perspective, the electronic ballot is suffering an advanced obsolescence process, with negative impacts on elections organization, particularly regarding the process ergonomics. In 1995, when the project was developed, it represented a compromise solution, balancing technical efficiency and costs trade-offs. At the time, the IBM/PC architecture was the best option, due to availability of compatible peripherals and accessories. Nevertheless, such architecture currently limits the ergonomics improvement to equipment operation, handling, maintenance and storage. After over fifteen years, other architectures have emerged emphasizing usability, autonomy, portability and security, which are the main requirements for the electoral process automation. These new devices allow many ergonomic improvements to the elections set up and logis-

\footnotetext{
* Corresponding author. E-mail: camargo.cr@gmail.com.
} 
tics of equipments transportation, thanks to their small sizes and low power consumption.

\section{Problem}

The Brazilian Electronic Ballot Box is too big, too heavy and has high-power consumption. This results in several ergonomic and logistics issues regarding its handling, storage and transportation. Its dimensions affect portability, which is considered a key ergonomic feature of the electoral process. Its transportation demands a complex logistics apparatus, involving a large amount of cargo vehicles. Its high-power consumption, in turn, limits its autonomy, leading to the creation of contingency structures in cases of power outages. During non-election periods, large structures are required for equipment storage, as well as complex and costly procedures for maintenance and internal battery recharge. Since there are more appropriate computing architectures, one can claim that the electronic ballot is currently obsolete and inadequate for the electoral process. The eventual adoption of equipment based on architectures such as tablets would allow significant ergonomic improvements, also probably having positive impact on costs.

\section{Technical requirements of the electronic ballot box}

The main requirements for the elections automation are: usability, portability, autonomy and security. The ergonomic simplicity in all operations of configuration, installation and use of the equipment are essential to the efficiency of the electoral process. The vast geographic coverage and capillarity of the elections, lead to concerns about portability and handling of the equipment. The limitations of infrastructure at the polling stations, in turn, require devices capable of working in varied conditions, even in the absence of external power sources. Finally, the computerized systems must ensure the integrity and confidentiality of the voting data stored in the equipment.

\subsection{Standalone device}

The current electronic ballot box has an implicit technical constraint that stems from a security assumption. This constraint implies on the device functioning isolated (standalone), without any built-in connectivity device (e.g. ethernet, wifi, $3 \mathrm{G}$ and so on).
The main concern was that any networking capabilities could be tampered with, thus making the whole electoral process less trustworthy.

This constraint was established in 1994, as part of the technical and functional requirements of the electronic ballot box. It's now quite evident that at the time there was a lack of data communication alternatives. By then the security mechanisms and tools to guarantee the integrity, security and secrecy of the traffic of the election very sensitive data were really incipient.

However, there are currently well-established data communication technologies capable of offering security levels up to par to automate most data transfer process necessary to the elections. Thus, with current communications protocols security levels, most of the data transfer taking place around the electronic ballot box could be automated. For instance, those activities related to the configuration, data upload, voting tally transmission, among others, could be highly simplified by wireless communication.

Thus, an important measure to enhance the electoral process is to allow the use of data communication features to improve data transfer. Besides the obvious agility gains (by speeding up data transfers), it is certain that these capabilities would enhance the whole process ergonomics, preventing blunders arising from activities that are now very prone to operational or media failure errors (e.g. read/write errors in the media used to transfer data to and from the ballot box). And, while this could be disputed, by adopting secure data communication protocols it is possible to achieve even higher levels of security than the current ones.

\subsection{Customized equipment}

In the early nineties, there were no COTS (commercially off-the-shelf) devices capable of fulfilling all the requirements to be used as the Brazilian electronic ballot box.

After long debates that took place in the Electoral Justice, a set of guidelines were defined on how to conceive and to build a customized device:

- The candidates are going to be identified by numbers;

- The voting device ("voter terminal") should have only a numeric keyboard, an abstention or blank vote key, and the control keys to correct a mistake and to submit the vote;

- The device should be physically monolithic; 
- To assure its autonomy ${ }^{2}$, it should be able to operate on conventional power outlets ( 90 to 240 volts), internal battery, and easily found external batteries, such as car batteries;

- Other functional and non-functional requirements.

The device design took into account constraints due to regional idiosyncrasies, a country with continental dimensions, with a great variety of operational conditions that could affect the device operation. There's no questioning that it was then the best way to make electronic voting a reality. However, after fifteen years, there is currently equipment largely available in the market suitable to be retooled for the electoral process.

One side effect of having a customized device is only being able to purchase it from a few suppliers. Only two companies manufactured Brazilian electronic ballot boxes - Unisys and Diebold/Procomp but since 2004 the latter is the sole supplier. Other companies lost interest in bidding as a result of the cumulative knowledge that the long time supplier has. It's quite difficult for another company to outplay the competitive advantage developed by Diebold.

Consequently, it is time to look for an alternative among available devices suitable to be retooled for the electoral process, because it will incur in purchasing costs reduction in the long run. The reliance on a sole supplier is undesirable, because it may lead to the conclusion that the prices charged are left undisputed by a healthier market logic.

\section{Logistics of the current electronic ballot box}

The physical attributes of the current electronic ballot box have major implications for the logistics of storage and transportation of the devices, particularly during elections. When in its standard case, the device has:

- Dimensions: $52.5 \mathrm{~cm} \times 42.5 \mathrm{~cm}$ x $24 \mathrm{~cm}$ (Width $\mathrm{x}$ Depth $\mathrm{x}$ Height)

- Volume: $0.05355 \mathrm{~m} 3$

- Weight: $9.8 \mathrm{~kg}$

2 The independent operation of the electronic voting machine has greatly evolved since the first models, as a consequence of reduced power consumption by the equipment and advances in battery technology.
For each State Electoral Court that adopts a centralized storage scheme (most of them ${ }^{3}$ ), the phases for device transportation and storage are:

- Storage of devices in between elections at the central storage;

- Device maintenance;

- Transportation of the ballot box from the central storage to the electoral zones;

- Transportation of the ballot box from the electoral zones to the voting places;

- Returning the ballot box from the voting places to the electoral zones;

- Returning the ballot box from electoral zones to the central storage.

Note that the ballot box is for the most part inactive, and it's going to be turned on (off election season) only to charge its internal battery, testing and corrective maintenance.

\subsection{Case study: State of Santa Catarina}

To illustrate what takes places we're going to break down the resources to handle electronic ballot boxes in the State of Santa Catarina:

- Quantity: 17,000 ballot boxes;

- Storage capacity: $910 \mathrm{~m} 3$

- Total weight: 166.7 tonnes

- Transportation from central storage to the electoral zones (and back): 19 trucks (mostly third axle vehicles), carrying an average of 900 ballot boxes each (48 cubic meters);

- Transportation from electoral zones to the voting places: 2,475 routes (collect and distribution), averaging almost 7 ballot boxes per vehicle ( 0.375 cubic meters).

Since the ballot box stockpiling at the end of the election day can be sluggish, some courts, including Santa Catarina's, put together a side logistics to gather the voting tally comprised of small vehicles and motorcycles to transport floppy disks and memory sticks.

\subsection{Extrapolation for the national scenario}

Expanding on a similar analysis to encompass the whole country, the resulting figures are superlative. There are currently 450,000 ballot boxes in operation; considering the same parameters of Santa Catarina, we obtain the following results:

3 The courts that hold the ballot boxes in electoral zones do not incur in transportation from and to the central warehouse. 
- Storage: 24,671 cubic meters;

- Total weight: 4,410 tonnes;

- Transportation from central storage to the electoral zones (and back): 512 trucks;

- Transportation from electoral zones to the voting places: 65,816 vehicles.

It is known, however, that the national scenario is even more complex than what we found in Santa Catarina. That state has privileged structural conditions, not to mention good infrastructure regarding public services. Other states have unique situations far more complex to be dealt with. Bigger states, such as São Paulo and Rio de Janeiro, have huge amounts of devices. Northern, center-northern and northeastern states need to move around smaller amounts of ballot boxes, but over much longer distances and on much precarious roads. Hence, the logistics difficulties for the transportation and storage of ballot boxes are even bigger for the national scenario than those of Santa Catarina and other southern states, which have a smaller area and better roads.

The large dimensions and weight of the current ballot box have a major impact on the logistics of the elections, incurring on greater costs for the whole operation. The physical attributes for the current ballot box deserve a meticulous analysis, aimed at simplifying the processes involved. Smaller and lighter electronic ballot boxes would imply in reduced costs for storage facilities and transportation capacity.

\section{Electronic ballot boxes operations}

The electronics of the current electronic ballot box has very limited computational capabilities. It's comprised of an adapted microcomputer with only two storage media types: floppy disks (or memory sticks ${ }^{4}$ ) and flash memory cards.

The main operations are:

- Self-testing and maintenance;

- Setup for the elections;

- Vote casting by electors; and

- Tallying votes and other voting data.

The above mentioned floppy disks or flash memory cards support the transfer of data for these operations. The dependency on these media makes the operations complex and time-consuming, besides making it necessary user interfaces to deal exclusively with those data exchanges. Additionally, the conver-

4 Memory sticks began to replace floppy disks in 2010 . sion from and to these media is a source of recurring operation errors.

\subsection{Self-testing and maintenance}

The self-testing is triggered by the activation floppy disk. While technically simple, this operation goal is to preserve the device integrity and identify any malfunctioning, and is of interest to the Electoral Justice.

\subsection{Setup for the elections}

Setting up the ballot boxes for an election consists of transferring data necessary to the voting (e.g, candidates data, voters, and so on). However, the process has the following steps:

- Media load: loading up the memory cards (setup media), with data for several electoral zones;

- Ballot box setup: setting up each ballot box with the setup media;

- Operation log: transferring setup information $\left(\operatorname{logs}\right.$ and assignment table ${ }^{5}$ ).

This is a very error prone process, as each operator handles dozens of memory cards at the same time. Some of those errors or omissions, for instance accidentally skipping the assignment table, have serious implications for the electoral process.

\subsection{Voting casting by electors}

On voting day, the person in charge of the election precinct (the site that features a ballot box) is supposed to:

- Verify each voter identity by checking their documents and matching signature on the attendance record;

- Once properly identified, the person in charge types the voter id on a small numeric keyboard to enable voting by that elector;

- When the ballot box gives positive feedback on the voter assignment to that election precinct and that she has not voted yet, the voter is allowed to proceed and cast the vote.

The current rules only allow voting by voters assigned to the specific election precinct. Electors away from their "electoral domicile" cannot cast votes, but

\footnotetext{
5 The assignment table associates the ballot data (e.g. serial number), details of polling stations and records the time of operation (timestamp); this table is published for the supervision by the political parties.
} 
can give a formal justification for not voting (voting is mandatory in Brazil).

\subsection{Tallying up voting data}

Each vote casted during Election Day is quickly tallied by the ballot box at the end of the voting period. However, these aggregated information (e.g. ballot box bulletin, attending and non-attending voters, operational log, and so on) have to come a long way until they get to the computational hubs in charge of vote tallying at each regional electoral court. Once the voting is over, the following processes take place:

- Electronic ballot box: transfers the voting results (among other files) to the floppy disk;

- Auditing and control personnel: insert the floppy disk (and accompanying documents) in a sealed envelope to assure data security and integrity;

- Transportation: the floppy disk envelope is sent to the electoral hub where it's data is uploaded to specific purpose computers (Transport System);

- Finally, after compiling data from several electoral zones, those are uploaded to the general tallying computers.

Some states diverge somehow from this process; the most common variation is setting up data transfer facilities at some voting places. This measure speeds up data transfer, but builds up costs in infrastructure (e.g. crew, computer, secure data connections, etc.).

\section{Requirements for a vote casting device}

Factors involved in an electoral process become complex due mainly to its grand scale. Official elections are single-day events that accommodate millions of electors. On the other hand, the vastitude of the Brazilian territory, with varying population density, transportation infrastructure and electricity grid capacity among other factors, contribute to increase sensibly the inherent complexity of election management. And, obviously, a great deal of power is at stake at the elections, where both representatives to the Congress and Executive are going to be chosen by the electors.

Some important vote casting device requirements stem from the above mentioned factors. The main ones are:

- Usability (ease of learning and of use): the device is used by millions of people from varying sociocultural backgrounds, thus it must be easily understood and operated;

- Portability: as the device is supposed to function all over the country, it must be easily transported, with emphasis on a single person being able to carry all the stuff to run a electoral precinct;

- Power autonomy: a single battery charge should last the voting period during the election day;

- Security: the device should support security protocols for secure data storage and transfer.

Ergonomics straightforwardness in all setup and usage activities is paramount to the electoral process efficiency. The elections geographical immensity and coverage make device portability and handling a major concern. A precarious infrastructure calls for robust devices capable, for instance, of functioning in the absence of an external power source. Finally, the resulting computerized system must assure the device-stored voting data integrity and secrecy.

The current device fails to fulfill ease of use - particularly those concerned in the device setup - and portability requirements. Its size and weight make it a burden to store and move around. Current tablet computers, on the other hand, are fully suitable to the process, besides featuring capabilities that may prove instrumental to the electoral process.

\section{Proposed solution}

In order to improve the electronic ballot box ergonomics aspects we devise a solution based on tablet computers. The proposed solution consists on replacing current equipment by tablet-based devices, because their technical characteristics best meet the requirements of the Brazilian electoral process, especially those related to portability and autonomy. They are small and low power consumption devices, which facilitates their logistics and autonomy. Likewise, they offer a wide range of built-in capabilities (e.g. touch screen, speaker and microphone), that enable highly usable and simple user interfaces. Finally, their operational systems features allow for the development of highly secure applications, suitable to the requirements of a voting process.

\subsection{Replacing current PC-based by tablet-based ballot boxes}

Tablets adoption is suitable to an incremental roll out strategy, adding new services and features to the electoral process. The device has capabilities that can 
be leveraged, but it can be done gradually. At first, it's only reasonable to devise a solution very similar to the current one. The device replacement alone would increase portability and reduce storage and transportation logistics. A later phase could concern devising new services that would better suit some electoral processes.

\subsection{Improvements to the setup procedure}

The setup consists of loading up the voters and candidates data for the device's assigned electoral zone. The adoption of a proper data communication network, for instance wifi, would simplify and improve this activity ergonomics. Simply put, the setup would consist on assigning the device to a electoral zone and precinct to trigger the automatic download of election-related data to the device (voters, running candidates, candidates portraits, political parties and so on) as well as operational and audit data (logs and the assignment table).

\subsection{Voting data transfer}

The intervening time from the end of the voting period to voting data tallying at the electoral zone is mostly due data transfer activities (e.g., writing data on floppy disks, sealing the envelope, etc.) and the transportation of those floppy disk containing envelopes to the data transfer hubs.

At those voting sites featuring data communications facilities (e.g. a landline or cell phone, wifi and such) the ballot box itself could be in charge of voting data upload (e.g. ballot box bulletin, attending and non-attending voters, operational $\log$, and so on) without human intervention.

Pending a more detailed research, it's known that the vast majority of Brazilians cast their votes in urban sites where telephony services are available. All but northern, center-northern and some regions in northeastern states have communications infrastructure that could be leveraged to support a voting data secure network (and those are the states where the majority of voters live).

Santa Catarina State, for instance, has $84 \%$ of its voters assigned to voting sites in urban areas. Those electors are assigned to $74 \%$ of the state electoral zones. That means that roughly $84 \%$ of votes could be uploaded and tallied up immediately after the end of the voting period. It also means that for only a quarter of the electoral zones some sort of logistics would have to be put in place to take the ballot boxes to a data transfer hub.

\section{Tablets capabilities and implications for voting}

Most tablet computers ${ }^{6}$ have native capabilities that could be leveraged to improve the electronic ballot box, particularly regarding its usability and security. The capabilities are enumerated bellow and whose potential use in the electoral process is briefly explored in the following sections:

- Capacitive touchscreen;

- Speakers (and headphones jack);

- Microphone;

- Gyroscope (three axes);

- Accelerometers;

- GPS (assisted);

- Camera (for photos or videos).

\subsection{Easy of use (usability)}

Although voting is already considered easy, a touch capable screen makes it possible to explore different interaction styles, based on direct manipulation and with greater directness (typing on a keyboard while looking at a screen is less direct that touching something on the screen). Besides the touchscreen, some other features could improve on ease of use:

- For the visually impaired, it is easy to adapt screen readers already in use with the current ballot box;

- The gyroscope could give feedback on whether the ballot box was properly positioned at the voting site (the right angle of inclination to facilitate visualization of the voter).

\subsection{Portability}

The main factors impacting portability are the tablets reduced dimensions and weight, particularly when compared to the current ballot box. These physical attributes afford a major simplification concerning transportation, with important ramifications for electoral precinct setup and management. At least in theory, a single person would be able to carry all the stuff to run an electoral precinct.

6 In this study, using the Apple iPad2 as reference. 


\subsection{Autonomy}

Current tablets are supposed to function 10 hours on a single charge. That should last for a whole voting period on Election Day (9 hours, from 8:00am to 5:00pm) but would not exempt safeguard measures, whether in the form of a power outlet or external batteries.

\subsection{Security}

Security-wise tablet capabilities may boost great improvements. GPS and accelerometer provide information on the ballot box undue displacement and meddling with. Alerts on unexpected or suspicious events could be issued. Bellow some of the envisioned possibilities are detailed:

- Keep tab of geographic placement (geo tagging), and compare it to known places where the device should be (typically, the electoral zone or the voting site). At a previously set interval, the device would log the positioning data (and optionally send it to a server);

- Alerts would be issued for devices detected outside their planned itinerary to allow the detection of suspicious handling;

- The accelerometer could be used to detect people meddling with the device at unusual periods of the day, for instance, late at night; that event would issue an alert containing the current device location;

- Networking capabilities could also be useful to act upon the device whenever suspicious activities are detected, rendering it a non-working ballot box unless some condition is restored, like the location being the same as the assigned electoral precinct (voting site).

There are also other resources that could be adopted for auditing purposes:

- Voice recording of relevant events at the electoral precinct;

- Photographs of poll workers and the administrator;

- Photographs of attending voters with non-valid identifications for further investigation.

\section{Electronic ballot box and voter authentication}

Voter authentication is the most crucial phase in the voting process, and precedes ballot activation. The current process is based on verifying voter bio- graphical information and her signature. It's considered deficient, faulty and prone to fraud as it depends on poll workers (which are ordinary citizens) being skillful and conscientious.

Since 2007 the Brazilian Electoral Justice is championing the construction of a voters biometrical database, to make possible fingerprint verification. Biometric authentication is considered to be secure, but it depends on the quality of voters fingerprint data gathered in the database and quality of recognition by the ballot box.

Most tablet devices commonly found do not feature biometric authentication, but they could be easily outfitted with biometric hardware and software.

\section{Ergonomics in the electoral process}

The ergonomics of all electoral process activities are of uppermost importance to successful elections. Cognitive ergonomics related usability requirements for the interaction of millions of people (voters and poll workers), many of them with low computer literacy, are fundamental. The directness and engagement of touch interfaces may prove beneficial to the users.

Similarly, the use of smaller devices would facilitate portability and reduce the number of people and vehicles involved with transportation and storage logistics, leading to significant improvements in physical and organizational ergonomics. Therefore, smaller and low power consumption equipment would lead to major improvements in the ergonomics of most of the elections processes.

\section{Conclusion}

The improvement of Brazilian electoral process depends on a thorough change in the current electronic ballot box architecture. The equipment in use limits ergonomics improvements, besides incurring in high logistics costs for transportation and storage, which can be substantially reduced by the adoption the tablet computers. The automation of elections in Brazil is one of the most successful cases of mass computerization, with worldwide recognition. However, after using this architecture for fifteen years, the technological equipment upgrade is crucial to solve the obvious logistics and ergonomics issues. 


\section{References}

[1] Acquisti A. Receipt-free homomorphic elections and write-in ballots. Cryptology ePrint Archive, Report 2004/105.

[2] David Chaum. Untraceable electronic mail, return addresses, and digital pseudonyms. Communications of the ACM, 24(2):84-88, 1981

[3] Atshushi Fujioka, Tatsuaki Okamoto, and Kazuo Ohta. A practical secret voting scheme for large scale elections. In Auscrypt '92, pages 244-251. Springer-Verlag, LNCS 718, 1992.

[4] Okamoto, Tatsuaki. Receipt-free electronic voting schemes for large scale elections. In Security Protocols Workshop, pages 25-35. Springer-Verlag, LNCS 1361, 1997.

[5] Chow, S.S.M., Liu, J.K., and Wong, D.S. Robust ReceiptFree Election System with Ballot Secrecy and Verifiability. In Proceedings of NDSS. 2008.

[6] Vanderheiden, G.C., Using extended and enhanced usability (EEU) to provide access to mainstream electronic voting machines, Information Technology and Disabilities, Vol. X, No. 2 (December 2004)

[7] Alvarez, R. M., Ansolabehere, S., Antonsson, E., Bruck, J., (2001) Voting - What Is, What Could Be.

[8] Quesenbery W., (2001) Voting and Usability: Lessons from the 2000 Presidential Election STC-PMC

[9] Event Handling Guide. Data Management: Event Handling. Apple Inc, Cupertino, CA. 2011-03-10. 